\title{
Anomalous Behaviour of the Electric Field in Highly-Compensated Non-Uniform Semiconductors at Low Temperatures
}

\author{
J.A. Jiménez-Tejada, A. Palma, A. Godoy and J.E. Carceller \\ Departamento de Electrónica y Tecnología de Computadores, Facultad de Ciencias, Universidad de \\ Granada, 18071 Granada, Spain
}

\begin{abstract}
The electric field and net charge density are simulated in a semiconductor structure with deep centers and a non-uniform dopant profile. Different regions of the electric field and net charge density are found in the device. The analysis of the distribution of these variables allows measurements devoted to the characterization of deep impurities at low temperatures to be interpreted.
\end{abstract}

\section{INTRODUCTION}

Due to the importance of defects, traps, and impurities in electronic devices such as Schottky diodes, pn junctions, and MESFETs, many combinations of deep-levels and semiconductor structures have been analyzed, with attention being paid recently to highly-doped semiconductors [1-3].

However, if the dopant atoms present in a semiconductor are non-uniform, and there is a high concentration of deep levels, then anomalous distributions of the electric field in the semiconductor can arise.

\section{DESCRIPTION OF THE STRUCTURE}

To demonstrate this distribution and the important effects that are produced, a reverse-biased gradual junction with two deep levels, an acceptor one in the upper half of the bandgap and a donor one in the lower half (the case of platinum in silicon), has been studied both numerically and experimentally.

The dopant profile here considered corresponds to a real sample and is shown in Figure 1, where two horizontal lines are drawn to indicate two values of the concentration of the deep levels and the points where these concentrations equal the dopant profile. To numerically analyze this structure we simultaneously solved the Poisson and Schockley-Read-Hall equations [4]:

$$
\begin{gathered}
\frac{d^{2} \Phi}{d x^{2}}=-\frac{q}{\epsilon_{s}}\left[p(x)-n(x)+N_{D}(x)-N_{A}(x)-N_{T} f_{T 1}(x)+N_{T 2}\left(1-f_{T 2}(x)\right)\right] \\
\frac{\partial f_{T}}{\partial t}=-\left(c_{n} n+c_{p} p+e_{n}+e_{p}\right) f_{T}+\left(n c_{n}+e_{p}\right),
\end{gathered}
$$


where $p(x)$ and $n(x)$ are the distributions of free holes and electrons respectively, $N_{D}-N_{A}$ is the shallowdopant profile, $N_{T 1}$ and $N_{T 2}$ are the deep-level densities (in our case $N_{T 1}=N_{T 2}$ ), $f_{T 1}$ and $f_{T 2}$ their occupation functions, $\mathrm{q}$ is the absolute value of the electron charge, $\epsilon_{\mathrm{s}}$ is the silicon-dielectric constant, $c_{n}, c_{p}$ are the capture coefficients, and $e_{n}, e_{p}$, the thermal-emission coefficients.

The solution of these equations gives the distribution of the potential along the junction, $\Phi(x)$, and from this the distribution of any other electrical magnitude such as the electric field and net charge density.

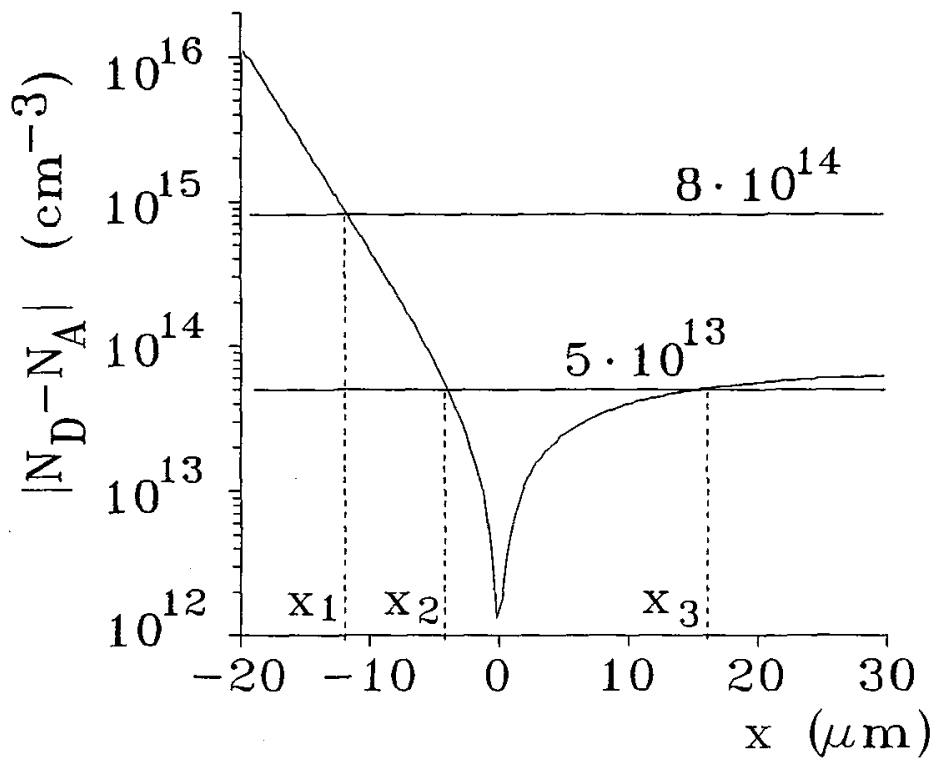

Figure 1: Shallow-dopant profile. p-region: $x<0$, n-region: $x>0$. Horizontal lines correspond to two values of deep level concentration.

\section{RESULTS}

In order to better understand this type of structure it is necessary to show the evolution of these magnitudes at low temperatures, especially since most of the methods for the characterization of deep levels take place in this range.

First, if we show the evolution of the electric field and charge density in a gradual $p^{+} n$ junction with a low concentration of deep levels, no extra information can be extracted from our numerical results: a higher value of the electric field at $x=0$ and a broadening of the transition region when the temperature decreases (the ionization factor increases), as shown in Figure 2. However, if the concentration of the deep level is increased or the temperature is diminished in not so highly doped samples, some anomalies appear. In Figures 3 and 4, the electric field and charge density are shown for these two types of samples respectively.

A new component of the electric field and a new distribution of charge displaced and separated from the known distributions located around the metallurgical junction can be seen. Note that the lower the temperature or the higher the concentrations of deep levels, the more acute this phenomenon. At a fixed temperature, the field value in the metallurgic junction $(x=0)$ is smaller with the presence of deep levels. Other maximums are placed far away from $x=0$ if the deep level concentration is high. However, this fact is not a direct consequence of the absolute value of the deep impurities but of the shallow-deep impurity concentration ratio. In fact, these extremes are located just at the point where the concentration of deep impurities equals the concentration of shallow ones (points $x_{I}, x_{2}$ and $x_{3}$ in the 

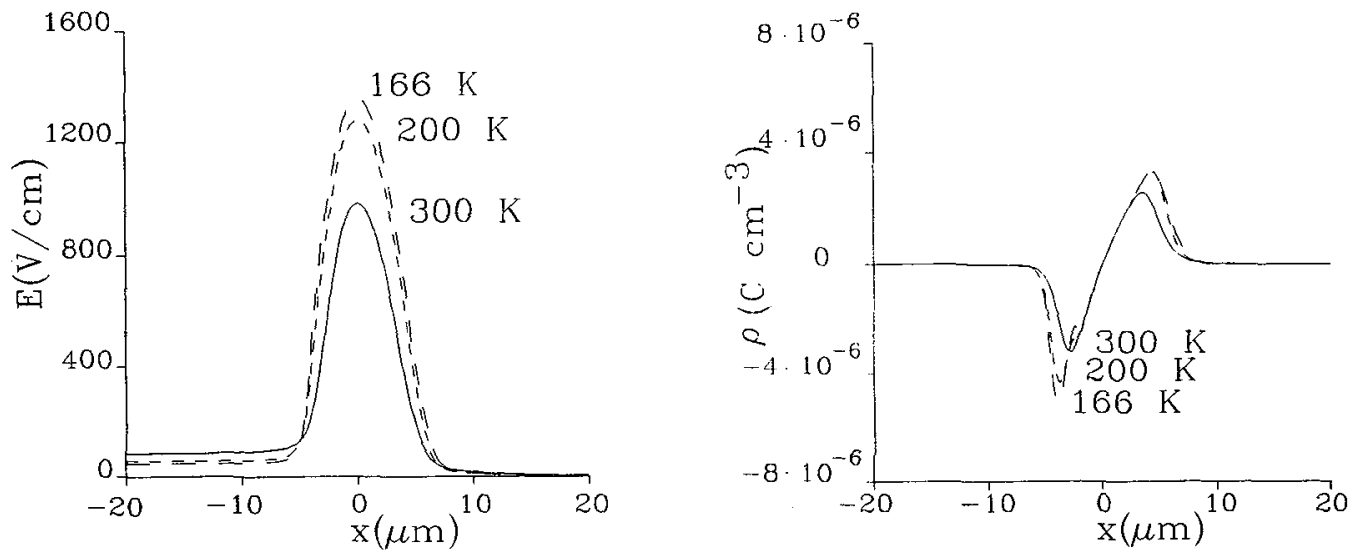

Figure 2:Electric field and net charge density in a gradual $\mathrm{p}^{+} \mathrm{n}$ junction with deep levels at $-0.2 \mathrm{~V}$. Donor concentration in n-region, $\mathrm{N}_{\mathrm{D}}=6.7 \cdot 10^{13} \mathrm{~cm}^{-3}$, deep-level concentration $\mathrm{N}_{\mathrm{T}}=10^{13} \mathrm{~cm}^{-3}$.
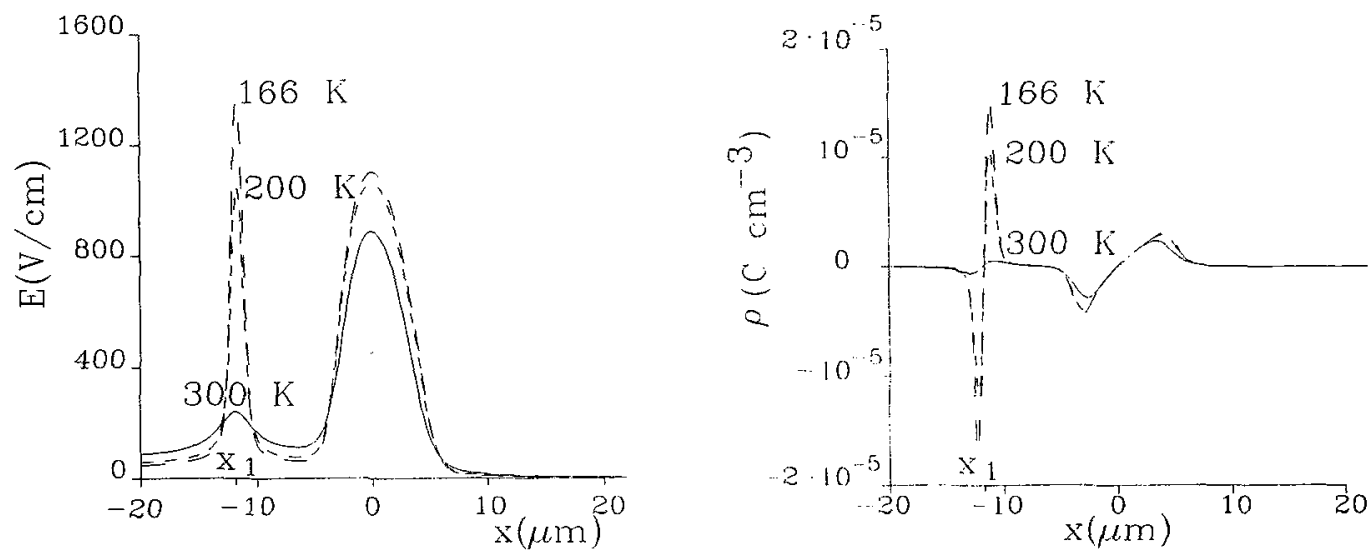

Figure 3: Electric field and net charge density in a gradual $\mathrm{p}^{+} n$ junction with deep levels at $-0.2 \mathrm{~V}$. Donor concentration in $n$-region, $N_{D}=6.7 \cdot 10^{13} \mathrm{~cm}^{-3}$, deep-level concentration $N_{T}=8 \cdot 10^{14} \mathrm{~cm}^{-3}$.
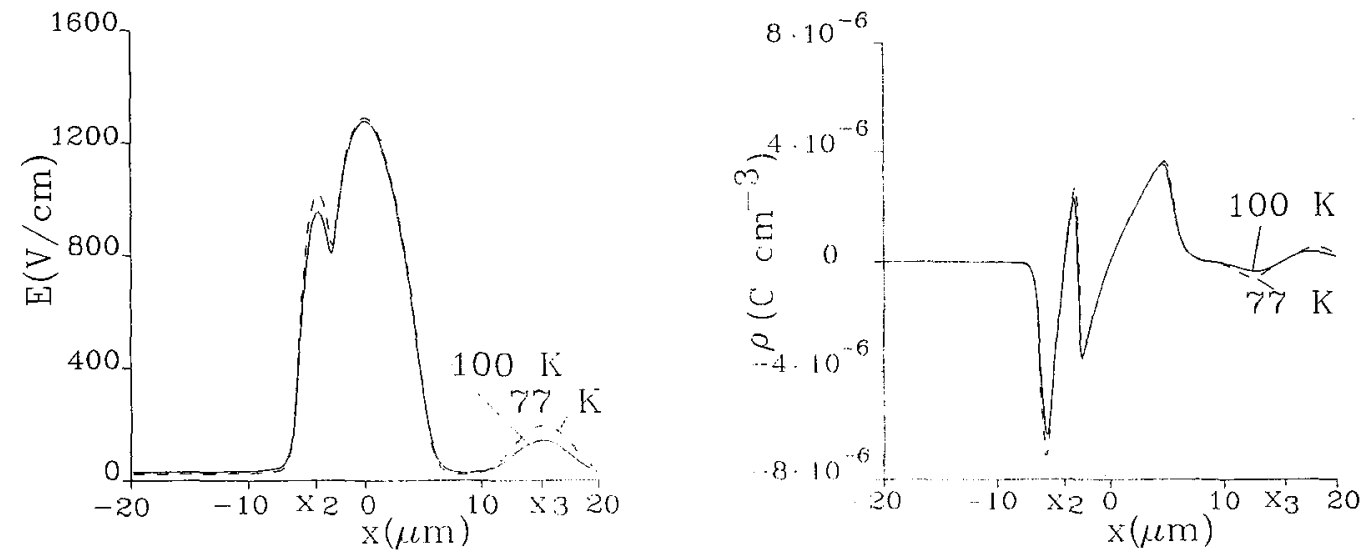

Figure 4: Electric field and net charge density in a gradual $p^{\top} n$ junction with deep levels at $-0.2 \mathrm{~V}$. Donor concentration in n-region, $\mathrm{N}_{\mathrm{D}}=6.7 \cdot 10^{13} \mathrm{~cm}^{-3}$, deep-level concentration $\mathrm{N}_{\mathrm{r}}=5 \cdot 10^{13} \mathrm{~cm}^{-3}$. 
figures). Therefore, the origin of these maximums is the non-uniformity of the dopant profile. These electric field maximums tend to disappear when the temperature is increased.

This fact is important when drawing the limits of the space charge region, as well as when interpreting results from measurements related to the space charge region. At high temperatures, the transition region is confined around the metallurgical junction and widens if an external reverse-voltage is applied, as is well known. However, at low temperatures the transition region is broadens due to the effect of the non-uniformity of the dopant profile and the higher ionization-factor of the deep levels. In this case, if the reverse-bias voltage is increased, the zones with a high field at the edges of the transition region prevent the width of this region from being modified (Figure 5). In the case where only one extra component of the electric field exists in one edge of the transition region (Figure 3), charge variations in the side where the fields lie cannot bring about a variation in the edge of this region but do so on the opposite edge. The reason for this phenomenon is the neutrality of charge. To maintain the neutrality in the whole transition region a variation of charge in the deep levels is cancelled with a modification of the transition region width. The p-transition region usually has to be increased due to an increment of negative charge inside it. However, if it is blocked, the n-transition region must decrease as though a drop of positive charge took place inside it.

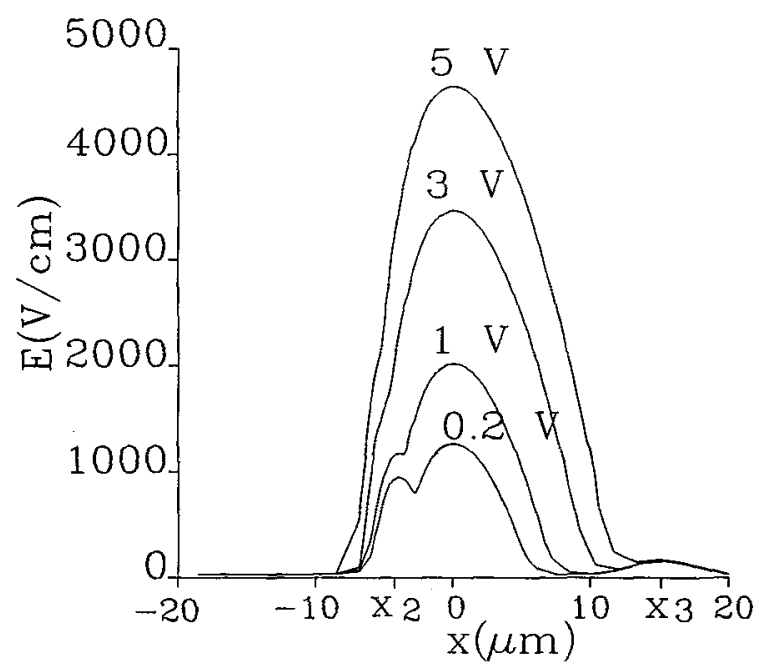

Figure 5: Electric field in a gradual $\mathrm{p}^{+} \mathbf{n}$ junction with deep levels at different reverse voltages at $100 \mathrm{~K}$. Donor concentration in n-region, $\mathrm{N}_{\mathrm{D}}=6.7 \cdot 10^{13} \mathrm{~cm}^{-3}$, deep-level concentration $\mathrm{N}_{\mathrm{T}}=5 \cdot 10^{13} \mathrm{~cm}^{-3}$. It can be seen how an increase of the field around the metallurgical junction does not broaden the width of the transition region due to the other two components of the field at the edges of this region.

An immediate consequence of this phenomenon is that the usual methods of characterization related to high-frequency capacitance measurements at low temperatures must be modified to take into account these non-uniformities. Under static characterization the freezing of high-frequency capacitance has to be taken into account. Under transient characterization the anomalous evolution of the borders of the transition region can lead to erroneous conclusions, which is the case for charge variations in majority or minority traps that, under a uniform profile, produce a variation in the edge of the transition region. However, in the structure we present here these charge variations do not necessarily produce a modification in the edge where the original variation is located.

As can be seen from Figures 3 and 4, the origin of these components of the electric field is the inversion of charge at the edges of the transition region. Due to the high ionization of deep levels in 
this part of the space charge region, the sample is locally overcompensated by the deep impurities.

To see the influence of this anomalous distribution and to quantify its effects, the high-frequency capacitance vs reverse voltage was obtained numerically and compared with experimental data $[5,6]$ in static and transient conditions. Among the effects that this new contribution of the electric field causes we noted: the freezing of high capacitance vs voltage at low temperatures, the disappearance of information on the deep level in the same range of temperatures (if no theoretical corrections are made), majority traps behaving like minority traps, and non-exponential constant-capacitance voltage transients when exponentiality is expected.

\section{CONCLUSIONS}

It has been shown that non-uniformity in the dopant profile of a semiconductor and the presence of a high concentration of deep impurities produce new components in the electric field and space regions that can alter the characterization of these types of samples at low temperatures.

\section{References}

[1] Andersson G.I., Engström O., J. Appl. Phys. 67 (1990) 3500-3510

[2] Stiévenard D., Lannoo M., Bourgoin J.C., Solid State Electron. 28 (1985) $485-492$

[3] Look D.C., Fang Z.Q., Sizelove J.R., J.Electron. Mater. 24 (1995) 1461-1464

[4] Jiménez-Tejada J.A., Lopez-Villanueva J.A., Cartujo P., Carceller J.E., Solid-St. Electron. 35 (1992) $1729-1736$

[5] Jiménez-Tejada J.A., Lopez-Villanueva J.A., Cartujo P., Vicente J., Carceller J.E., J. Appl. Phys. 72 (1992) 4946-4953

[6] Palma A., Jiménez-Tejada J.A., Banqueri J., Cartujo P., Carceller J.E., J. Appl. Phys. 74 (1993) 2605-2612 